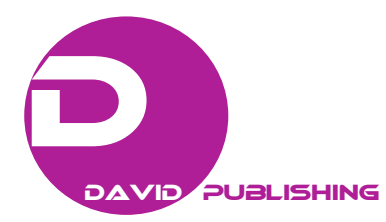

\title{
Enterohaemorrhagic Escherichia coli (E.coli 0157): A Case Report from Nepal
}

Ranjit Sah, Shusila Khadka, Neha Shrestha, Mahesh Adhikari, Hari Prasad Kattel, Niranjan Prasad Shah, Keshab Parajuli, Jeevan Bahadur Sherchand, Basista Rijal and Bharat Mani Pokhrel

Department of Microbiology, Tribhuvan University Teaching Hospital (TUTH), Institute of Medicine, Tribhuvan University, Kathmandu 44600, Nepal

\begin{abstract}
E.coli $\mathrm{O} 157$ is one of the important causes of bloody diarrhea which can be self-limiting or may complicate into Haemolytic Ureaemic Syndrome (HUS). We are reporting a case of Enterohaemorrhagic Escherichia coli (E.coli O157) in a thirty year old male who presented to the surgical OPD of Tribhuvan University Teaching Hospital (TUTH), Kathmandu, Nepal with the complaint of bloody diarrhea and abdominal pain along with the history of consumption of food in local restaurant. Stool sample of the patient was processed to find out the cause of bloody diarrhea. Microscopic examination of the stool sample revealed plenty of red blood cells but no pus cells. Stool culture on MacConkey agar revealed lactose fermenting (pink) colonies whereas on sorbitol MacConkey agar, sorbitol fermenting (pink) and non-sorbitol fermenting (pale) colonies were isolated. Both the sorbitol fermenting and non-fermenting isolates were identified as Escherichia coli on the basis of biochemical tests. The non-sorbitol fermenting colonies which were identified as Escherichia coli were serotyped which were positive on polyvalent antisera 3 and monovalent antisera O157. Therefore, on the basis of biochemical reaction and serotyping, Escherichia coli O157 (Enterohaemorrhagic E. coli) was identified as the cause of the bloody diarrhea. The patient was managed symptomatically without any antibiotics and got improved in few days without any complication.
\end{abstract}

Key words: EHEC, E. coli O157, Bloody diarrhea, TUTH, Nepal.

\section{Background}

Enterohaemorrhagic Escherichia coli (E. coli O157:H7) is a human pathogen responsible for outbreak of bloody diarrhea [1]. In most of the cases, it is self-limiting. But in some cases, it can progress to Haemolytic Ureaemic Syndrome (HUS) [2] and use of antibiotics further degrades the condition [1]. The term Enterohaemorrhagic E. coli (EHEC) is applied to those shiga toxin-producing $E$. coli (STEC) serotypes that have the same clinical, epidemiological and pathogenic features associated with the prototype strain E. coli O157:H7 [2]. The classic illness caused by Enterohaemorrhagic E. coli produces a watery diarrhea that progresses to bloody diarrhea with abdominal cramps and low-grade fever. The stool contains no leucocytes, which distinguishes from dysentery caused

Corresponding Author: Ranjit Sah, M.D., research field: microbiology (parasitology and bacteriology). by Shigella species and Enteroinvasive E. coli. The infection is potentially fatal, especially in young children and elderly adults [3]. The ideal method of identification of E. coli $\mathrm{O} 157: \mathrm{H} 7$ is isolation of nonsorbitol fermenting colonies on sorbitol MacConkey agar from a bloody stool with no leucocytes on microscopic examination $[3,4]$. We report a case of thirty year old male who presented to surgical OPD of TUTH with the complain of diarrhea and abdominal pain along with the history of consumption of food in local restaurant. Initially the diarrhea was watery but on later days became bloody. On palpation, the abdomen was soft but diffusely tender with hyperactive bowel sounds. To our knowledge, this is the first case of detection and isolation of E. coli $\mathrm{O} 157$ from Nepal.

\section{Method}

The patient was referred to microbiology laboratory 
of TUTH to rule out infectious cause. Stool sample was collected in a sterile container (Fig. 1) and processed for macroscopic and microscopic examination. Culture was done on MacConkey agar and sorbitol MacConkey agar simultaneously and incubated overnight at $37^{\circ} \mathrm{C}$ aerobically as microscopic examination showed plenty of RBCs but no pus cells which is usually a feature of diarrhea due to Enterohemorrhagic E. coli [3, 4]. On sorbitol MacConkey agar, sorbitol fermenting and non-fermenting colonies were found which were further processed for biochemical tests like oxidase, catalase, Hugh and Leifson oxidative-fermentative (OF) test, triple sugar iron agar test, indole production, Simmon's citrate utilization, urease production, methyl red test, Voges-Proskauer test, decarboxylation of arginine, lysine and ornithine [5, 6]. Since, the non-sorbitol fermenting colonies were identified as Escherichia coli, serotyping was done according to the instruction manual of Denka Seiken Co., Ltd. (Tokyo, Japan) by slide agglutination method with polyvalent antisera (1-8) followed by monovalent antisera (O18, O114, O142, O151, O157, O158) [7].

\section{Result}

Stool sample of the patient on macroscopic examination was loose and reddish (bloody) with no characteristic odour. Microscopic examination showed plenty of red blood cells but no pus cells. Stool culture of the patient on MacConkey agar revealed lactose fermenting (pink) colonies whereas on sorbitol MacConkey agar, sorbitol fermenting (pink) and non-sorbitol fermenting (pale) colonies were isolated (Figs. 2 and 3). On biochemical testing, the isolates were catalase positive, oxidase negative, fermentative on OF test, fermented glucose and lactose on triple sugar iron agar with gas production, indole test was positive and the organism was motile, citrate utilization and urea hydrolysis test were negative, methyl red test was positive and the organism decarboxylated lysine. On the basis of these biochemical tests (Fig. 4), both the isolates were identified as Escherichia coli phenotypically. The non-sorbitol fermenting colonies which were identified as Escherichia coli were serotyped which were positive on polyvalent antisera 3 and monovalent antisera O157 (Fig. 5). Therefore, on the basis of biochemical reaction and serotyping, Escherichia coli $\mathrm{O} 157$ (Enterohaemorrhagic E. coli) was isolated and identified. The patient was managed symptomatically without any antibiotics and got improved after few days without any complication.

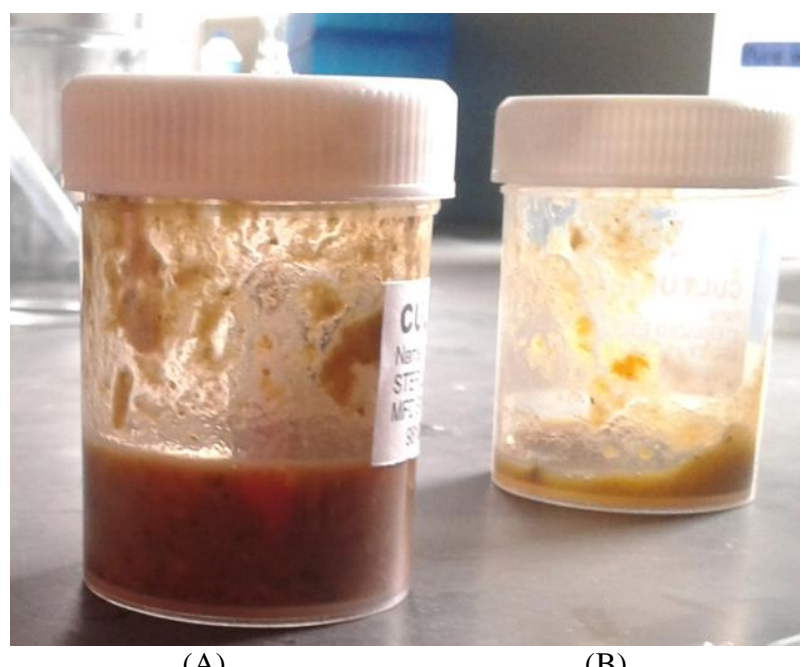

Fig. 1 Bloody Stool sample of the patients (A) in comparison to normal stool of the healthy individual (B).

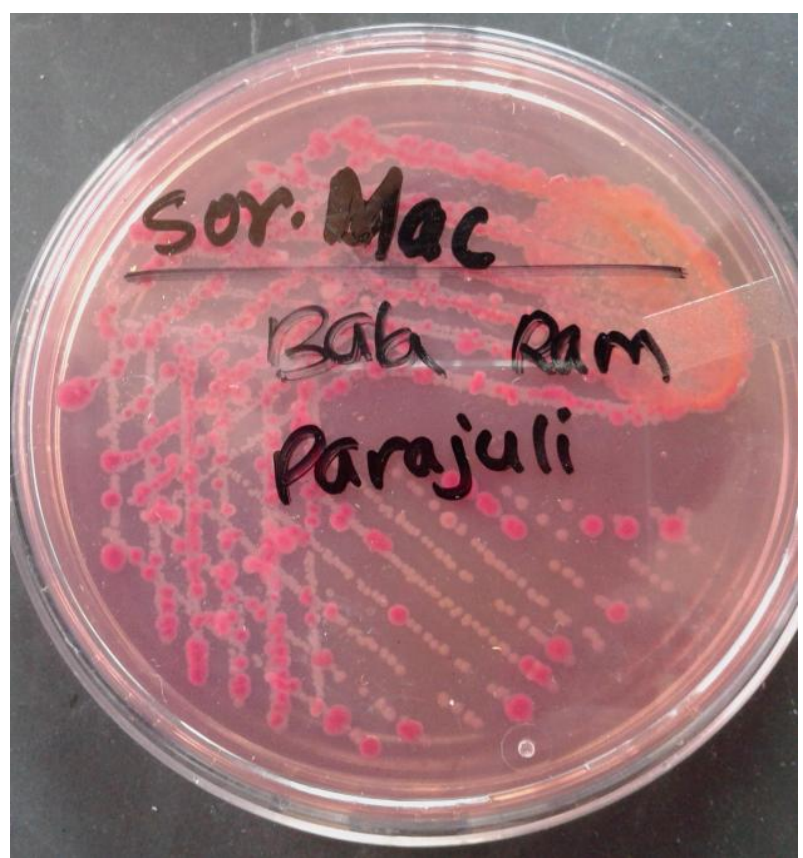

Fig. 2 Sorbitol fermenting and non-fermenting colonies of Escherichia coli on sorbitol MacConkey agar. 


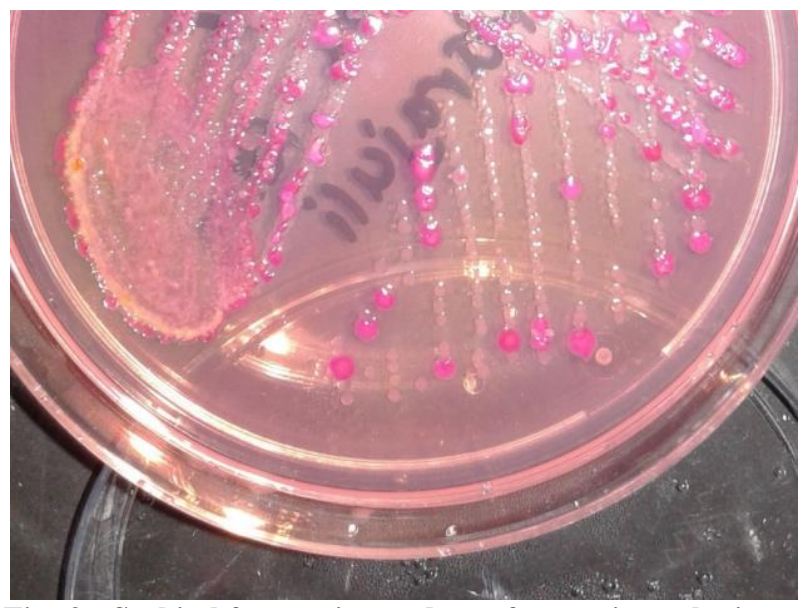

Fig. 3 Sorbitol fermenting and non-fermenting colonies of Escherichia coli on sorbitol MacConkey agar.

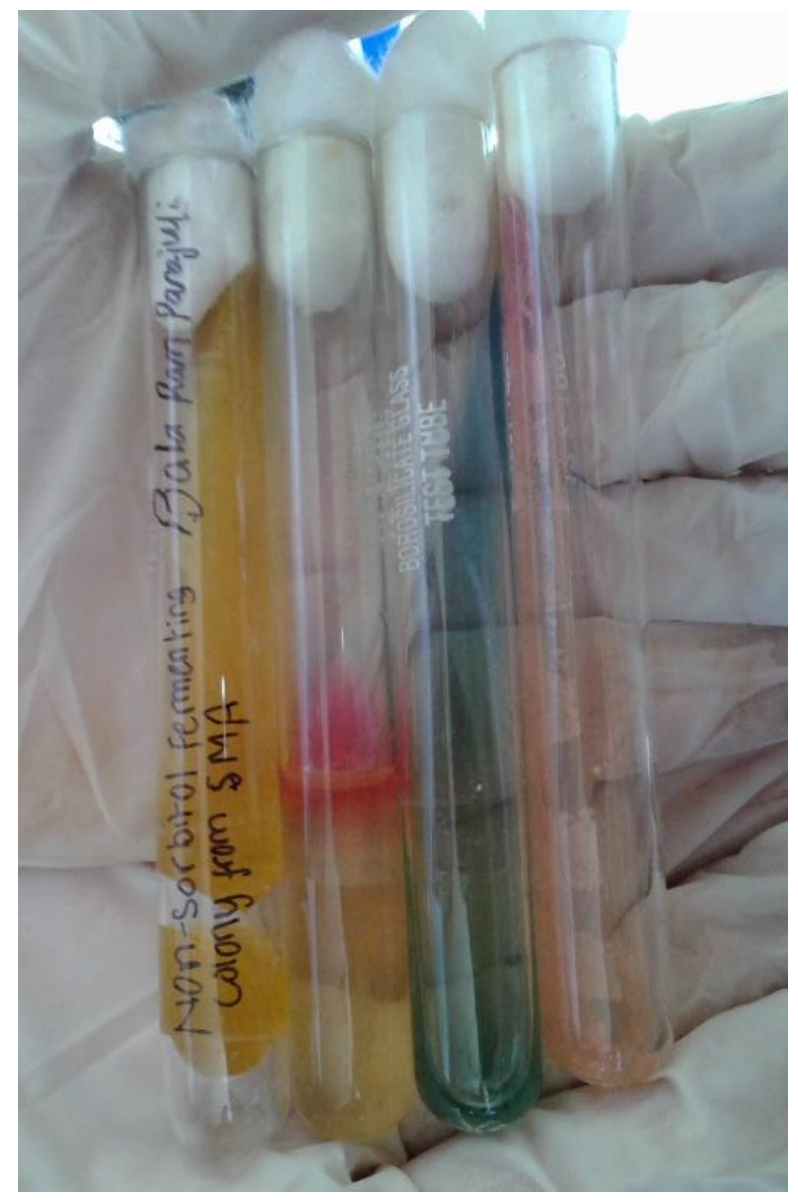

Fig. 4 Biochemical test of Escherichia coli.

\section{Conclusion}

E. coli $\mathrm{O} 157$ is an important bacterial pathogen to cause bloody diarrhea and is of public health importance as it can cause an epidemic outbreak. So stool sample of patient having bloody diarrhea should
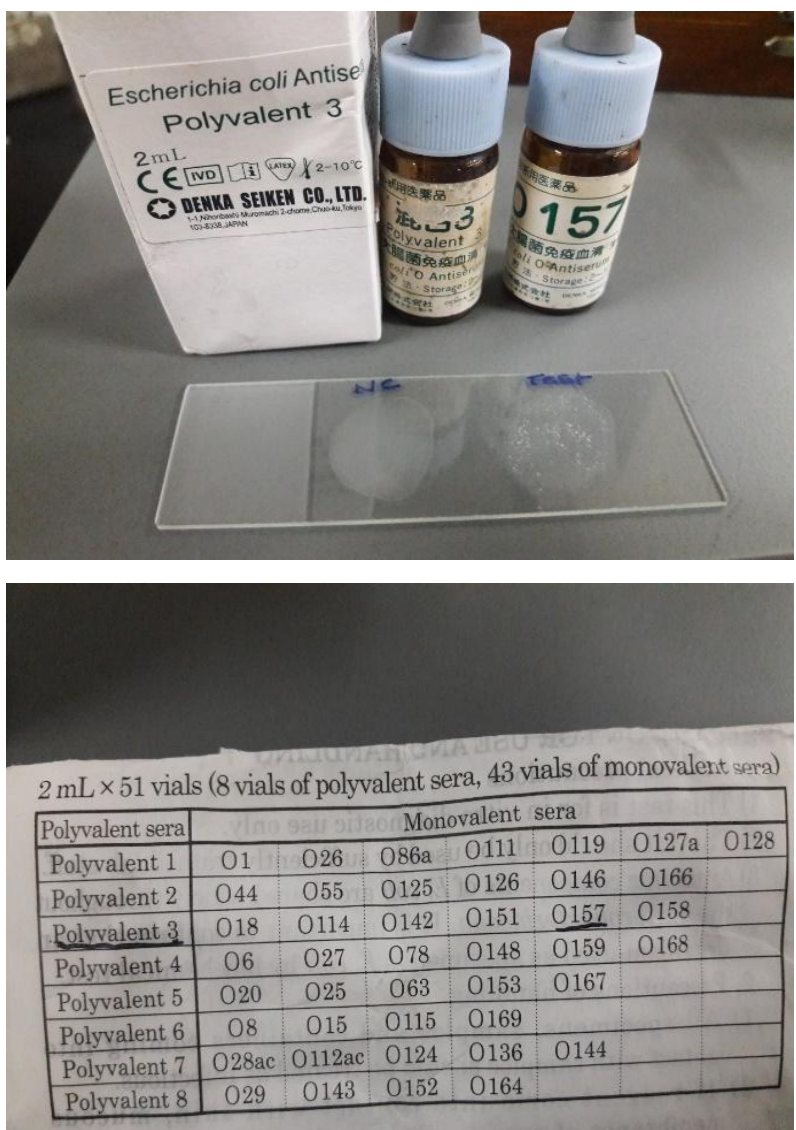

Fig. 5 Serotyping of Escherichia coli showing positivity (agglutination) with polyvalent 3 followed by monovalent 0157.

be cultured simultaneously on MacConkey and sorbitol MacConkey agar in routine laboratory to detect E. coli O157. As undiagnosed cases of bloody diarrhea caused by EHEC may lead to antibiotic therapy which may further precipitate HUS and cost the life of the patients, diagnosis is important for patient management and prevention of complications.

\section{References}

[1] Nguyen, Y., and Sperandio, V. 2012. "Enterohaemorrhagic E.coli (EHEC) Pathogenesis." Frontiers in Cellular and Infection Microbiology 2: 90.

[2] Jafari, A., Aslani, M. M., and Bouzari, S. 2012. "Escherichia coli: A Brief Review of Diarrheagenic Pathotypes and Their Role in Diarrheal Diseases in Iran." Iranian Journal of Microbiology 4 (3): 102-17.

[3] Mahon, C. R., Lehmanm D. C., and Manuselism G. 2014. Textbook of Diagnostic Microbiology, 5th edition. Missouri: Elsevier, 426-7.

[4] Esumeh, F. I., Isibor, J. O., and Egbagbe, I. D. S. 2011. "Screening for Escherichia coli O157:H7 in Diarrheic 
Patients in Benin City, Nigeria." J. Microbiol. Biotech. Res. 1 (4): 1-4.

[5] Khosravi, A. D., Khaghani, S., Sheikh, A. F., Ahmadzadeh, A., and Shamsizadeh, A. 2016 "Prevalence of Escherichia coli O157:H7 in Children with Bloody Diarrhea Referring to Abuzar Teaching Hospital, Ahvaz, Iran." Journal of Clinical and Diagnostic Research 10 (1): 13-5.
[6] Isenberg, H. D. 2007. Clinical Microbiology Procedures Handbook, 2nd Edition Update Wasington DC: ASM press.

[7] Erdogan, H., Erdogan, A., Levent, B., Kayalı, R., and Arslan, H. 2008. "Enterohemorrhagic Escherichia coli O157:H7: Case Report." The Turkish Journal of Pediatrics 50 (5): 488-91. 